\title{
Screening Masses and Improvement in Pure SU(2) Lattice Gauge Theory at High Temperatures
}

\author{
U. M. Heller ${ }^{\mathrm{a}}$, F. Karsch ${ }^{\mathrm{b}}$ and J. Rank*a,b \\ aSCRI, Florida State University, Tallahassee, FL 32306-4130, USA \\ bFakultät für Physik, Universität Bielefeld, P.O. Box 100131, D-33501 Bielefeld, Germany
}

From the long-distance behaviour of gluon and Polyakov loop correlation functions we extract masses resp. energies in the electric and magnetic sectors. We discuss their dependence on the temperature and on the momentum as well as the relevance of an improvement of the lattice discretization of the action.

\section{Introduction}

The high temperature plasma phase of QCD is characterized by the occurrence of chromoelectric and -magnetic screening masses $\left(m_{e}\right.$ and $m_{m}$ ) which control the infrared behaviour of the theory [1]. It has been known for a long time that the lowest order perturbative result for the electric mass in pure gauge theory is $m_{e, 0}(T)=$ $\sqrt{N_{c} / 3} g(T) T$. This is sufficient to cure infrared divergences of $\mathcal{O}(g T)$. The magnetic mass is known to be entirely of non-perturbative origin, as all orders in perturbation theory would contribute equally. However, a dependence of the form $m_{m} \sim \mathcal{O}\left(g^{2} T\right)$ is widely believed and would cure the higher order infrared divergences of $\mathcal{O}\left(g^{2} T\right)$. If one finds indeed $m_{m} \neq 0$, than $m_{m}$ contributes in next-to-leading order perturbation theory to $m_{e}$ [2],

$$
\begin{aligned}
& m_{e}^{2}(T)=m_{e, 0}^{2}(1+ \\
& \left.\quad \frac{\sqrt{6}}{2 \pi} g(T) \frac{m_{e}}{m_{e, 0}}\left[\log \frac{2 m_{e}}{m_{m}}-\frac{1}{2}\right]+\mathcal{O}\left(g^{2}\right)\right) .
\end{aligned}
$$

We note that if $m_{m} \sim \mathcal{O}\left(g^{2} T\right)$ the next-to-leading order correction to $m_{e}$ is $\mathcal{O}(g \ln g)$.

The above discussion shows that non perturbative methods are needed to obtain results not only for the magnetic but also for the electric mass. In the following we will present results for $m_{e}$ and $m_{m}$ that we calculated in $\mathrm{SU}(2)$ lattice gauge theory, using both the Wilson action and a tree-level

\footnotetext{
${ }^{*}$ Talk presented at the Lattice 97 conference by J.R.
}

Symanzik improved action with a planar 6-link loop. Simulations have been performed on lattices of sizes $32^{3} \times 4$ and $32^{2} \times 64 \times 8$ at temperatures above the critical temperature of the deconfinement phase transition from $T=2 T_{c}$ up to very high temperatures, $T \simeq 10^{4} T_{c}$, in order to get in contact with perturbative predictions for $m_{e}$.

\section{Screening Masses from Gluon Correla- tion Functions}

It was shown in [3] that the pole mass definition of the screening masses,

$m_{\mu}^{2}=\Pi_{\mu \mu}\left(p_{0}=0, \vec{p}^{2}=-m_{\mu}^{2}\right)$,

is gauge invariant although the gluon polarization tensor $\Pi_{\mu \mu}$ itself is a gauge dependent quantity. These pole masses can be obtained from the long distance behaviour of momentum dependent gluon correlation functions in the static sector $\left(p_{0}=0\right)$,

$\tilde{G}_{\mu}\left(p_{\perp}, x_{3}\right)=\left\langle\operatorname{Tr} \tilde{A}_{\mu}\left(p_{\perp}, x_{3}\right) \tilde{A}_{\mu}^{\dagger}\left(p_{\perp}, 0\right)\right\rangle$

with the momentum dependent gauge fields

$\tilde{A}_{\mu}\left(p_{\perp}, x_{3}\right)=\sum_{x_{0}, x_{\perp}} e^{i x_{\perp} p_{\perp}} A_{\mu}\left(x_{0}, x_{\perp}, x_{3}\right)$.

We define $p_{\perp} \equiv\left(p_{1}, p_{2}\right), x_{\perp} \equiv\left(x_{1}, x_{2}\right)$.

As $\tilde{G}_{\mu}$ is gauge dependent one has to work in a fixed gauge, which is in our case the Landau gauge. Details on the gauge fixing algorithm can be found in $\llbracket$. The relation between $\tilde{G}_{\mu}$ and $m_{\mu}$ 


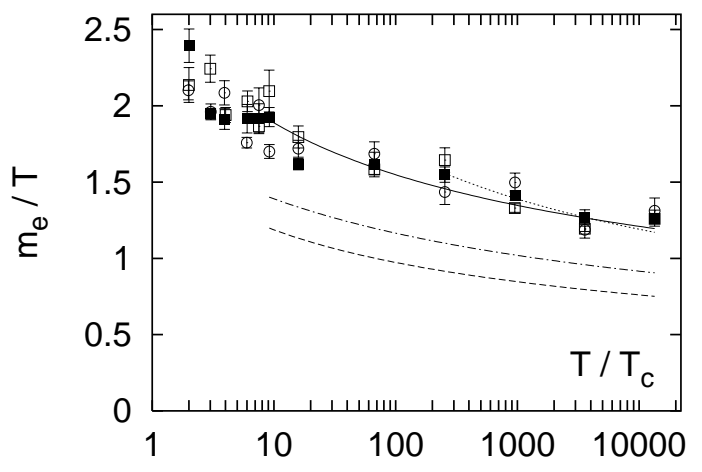

Figure 1. Electric screening mass in units of the temperature vs. $T / T_{c}$; see text for details.

and techniques how to extract $m_{\mu}$ most reliable from a lattice simulation are discussed in [5].

In Fig. 1 we show the electric screening mass in units of the temperature vs. $T / T_{c}$. The Wilson action data are symbolized by filled $\left(N_{\tau}=8\right)$ resp. open squares $\left(N_{\tau}=4\right)$, the data based on the treelevel Symanzik improved action with $N_{\tau}=4$ by open circles.

Within statistical errors, all these data are compatible. Especially the fact that an improvement of the action does not shift the data in any direction is a first hint that the electric mass indeed is entirely dominated by low momentum modes. A comparison of the data with the lowest order perturbative prediction (the dashed line) shows that this is not a good description although the functional dependence on the temperature does seem to describe the data well. For $T \geq 9 T_{c}$ we performed a one parameter fit to our data with the ansatz $m_{e}^{2}(T)=A_{\text {fit }} g^{2}(T) T^{2}$, using the twoloop $\beta$-function for the running coupling. The result $A_{\text {fit }}=1.69(2)\left(\right.$ with $\chi^{2} /$ dof $\left.=4.51\right)$, which is shown as a solid line in Fig. 1, exceeds the perturbative value $2 / 3$ by a factor of more than 2.5 .

To test the next-to-leading order result (11) we also calculated the magnetic mass $m_{m}$. We did this for the Wilson action with $N_{\tau}=8$. Our results for the ratio $m_{e}^{2} / m_{m}^{2}$ are shown in Fig. 2. They suggest that the ratio runs with the temperature as expected, $m_{e}^{2} / m_{m}^{2} \sim g^{-2}(T)$. A fit over the entire temperature range yields $m_{e}^{2} / m_{m}^{2}=$ $7.43(27) g^{-2}(T)$. The quality of the fit becomes

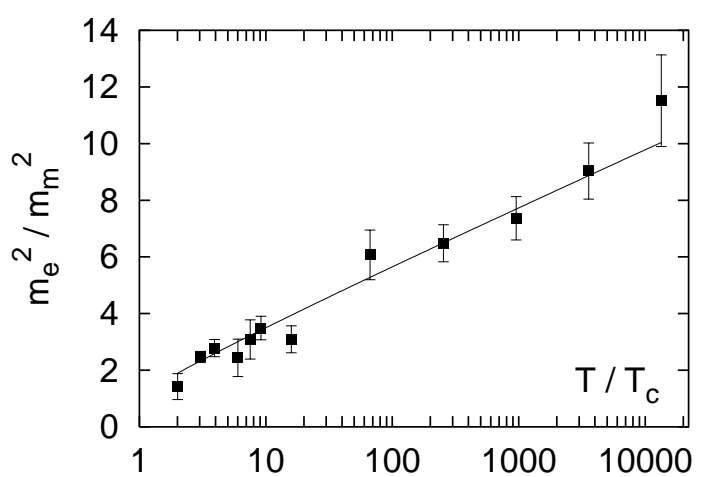

Figure 2. Squared ratio of electric and magnetic screening masses vs. $T / T_{c}$ on a $32^{2} \times 64 \times 8$ lattice using the Wilson action.

obvious from the small value of $\chi^{2} /$ dof $=1.39$.

Based on this result we fitted the magnetic mass itself, using the ansatz $m_{m}(T) \sim g^{2}(T) T$. In the temperature range above $3 T_{c}$ we obtain $m_{m}(T)=0.457(6) g^{2}(T) T$ with $\chi^{2} /$ dof $=1.50$. This is in good agreement with our result obtained in [4] for $T<20 T_{c}$.

With the numerical result for $m_{e} / m_{m}$ we are now able to compute $m_{e}$ in next-to-leading order, using Eq. (11). The result is shown by the dasheddotted line in Fig. 1. It lies about $20 \%$ above the tree-level result. However, it is still too small to describe the data. Therefore we have performed another fit of the electric mass, using the ansatz

$$
\begin{gathered}
\left(\frac{m_{e}(T)}{T}\right)^{2}=\frac{2}{3} g^{2}(T)\left(1+\frac{\sqrt{6}}{2 \pi} g(T) .\right. \\
\left.\left[\log \frac{2 m_{e}}{m_{m}}-\frac{1}{2}\right]\right)+B_{\text {fit }} g^{4}(T) .
\end{gathered}
$$

As the $g^{4}$ correction term leads to a temperature dependence which is too strong within the entire $T$-interval we have restricted the fit to very high temperatures, $T \geq 250 T_{c}$. The fit gives $B_{\text {fit }}=0.744(28)$ with $\chi^{2} /$ dof $=4.55$ (dotted line in Fig. 11).

So far we have only discussed the screening masses which had been extracted from zero momentum correlation functions. In addition, we also measured gluon correlation functions at finite momenta $p_{1} a=2 \pi k_{1} / N_{1}$ with $k_{1}=1,2$. In order to analyze modifications of the free particle 
dispersion relation, which arise from interactions in a thermal medium, we introduce a parameter $\kappa$ in the dispersion relation:

$\sinh ^{2} \frac{a E_{e}\left(p_{1}\right)}{2}=\sinh ^{2} \frac{a m_{e}}{2}+\kappa \sin ^{2} \frac{a p_{1}}{2}$.

For $m_{e}$ we use the results from the $\vec{p}=0$ measurements. For $T \rightarrow \infty$ one expects that the dispersion relation approaches that of a free particle, i.e. $\kappa \rightarrow 1$. In the temperature interval analysed by us, however, we do not observe any statistically significant increase in $\kappa$. We therefore only quote a value averaged over the temperature interval $T \geq 9 T_{c}$. We obtain $\kappa=0.37(10)$ for $k_{1}=1$ and $\kappa=0.65(3)$ for $k_{1}=2$.

\section{Polyakov Loop Correlation Functions}

For temperatures above $T_{c}$ the relation between the electric (or Debye) screening mass and the colour singlet potential $V_{1}$ is in lowest order perturbation theory given by [6]

$V_{1}(R, T)=-g^{2} \frac{N_{c}^{2}-1}{8 \pi N_{c}} \cdot \frac{e^{-m_{e}(T) R}}{R}$

which is again valid only at large distances. On the lattice one can extract $V_{1}$ by measuring Polyakov loop correlation functions

$e^{-V_{1}(R, T) / T}=2 \frac{\left\langle\operatorname{Tr}\left(L(\vec{R}) L^{\dagger}(\overrightarrow{0})\right)\right\rangle}{\langle|L|\rangle^{2}}$.

In addition, we are using not only $V_{1}$ to extract $m_{e}$ but also $V_{1 \text {,sum }}$ which is based on Polyakov loops that are averaged over hyperplanes $[5]$.

The numerical values for $m_{e}$ using this methods agree within errors with the data obtained from the gluon correlation functions at zero momentum. The values of the fits of $m_{e} / T$ using the two fit ansätze described in the last section are listed in [5].

To check whether or not an improvement of the action weakens the violation of the rotational symmetry caused by the lattice discretization we measured $V_{1} / T$ both along an axis, labeled with $(1,0,0)$, and along three different off-axis directions, $(1,1,0),(1,1,1)$, and $(2,1,0)$.

For each action we computed the fit of the $(1,0,0)$ data from which we calculated the $\chi^{2}$ deviation of the off-axis data. For all 12 temperatures we observed much larger deviations in the case of Wilson action than for the tree-level Symanzik improved action. In addition, the parameters of the fits of the $(1,0,0)$ data themselves have bigger errors and furthermore the fits have larger $\chi^{2}$ and lower goodness in the case of Wilson action than for the improved action.

\section{Summary}

We have studied electric and magnetic screening masses obtained from Polyakov loop and gluon correlation functions in the high temperature deconfined phase of $\mathrm{SU}(2)$ lattice gauge theory, using both the standard Wilson action and a tree-level Symanzik improved action.

For $m_{m}$ we find the expected $\mathcal{O}\left(g^{2} T\right)$ behaviour, $m_{m}(T)=0.457(6) g^{2}(T) T$. We also find $\left(m_{e} / m_{m}\right)^{2} \sim g^{-2}$, which suggest that the temperature dependence of $m_{e}$ is well described by $m_{e} / T \sim g(T)$ as expected by lowest order PT. On a quantitative level we do, however, find large deviation. Our result $m_{e}(T)=\sqrt{1.69(2)} g(T) T$, deviates strongly from the lowest order PT prediction, and it can only be insufficiently cured by the next-to-leading order term.

The improvement of the action does not show, within statistical errors, any significant modification in the behaviour of the screening masses, although we find that the violation of the rotational symmetry of the singlet potential, which also is used to extract $m_{e}$, is weakened.

\section{REFERENCES}

1. A.D. Linde, Phys. Lett. B96 (1980) 289.

2. A.K. Rebhan, Phys. Rev. D48 (1993) R3967 and Nucl. Phys. B430 (1994) 319.

3. R. Kobes, G. Kunstatter, A. Rebhan, Phys. Rev. Lett. 64 (1990) 2992 and Nucl. Phys. B355 (1991) 1.

4. U.M. Heller, F. Karsch and J. Rank, Phys. Lett. B355 (1995) 511.

5. U.M. Heller, F. Karsch and J. Rank, BI-TP 97/36, September 1997.

6. L.D. McLerran and B. Svetitsky, Phys. Rev. D24 (1981) 450. 